\title{
On Tachibana and Vishnevskii Operators Associated with Certain Structures in the Tangent Bundle
}

\author{
Lovejoy S. Das', Mohammad Nazrul Islam Khan² \\ ${ }^{1}$ Department of Mathematics, Kent State University, Tuscarawas, New Philadelphia, OH, USA \\ ${ }^{2}$ Department of Computer Engineering, College of Computer, Qassim University, Buraidah, KSA \\ Email: ldas@tusc.kent.edu,m.nazrul@qu.edu.sa,mnazrul@rediffmail.com
}

How to cite this paper: Das, L.S. and Khan, M.N.I. (2018) On Tachibana and Vishnevskii Operators Associated with Certain Structures in the Tangent Bundle. Journal of Applied Mathematics and Physics, 6, 1968-1978.

https://doi.org/10.4236/jamp.2018.610168

Received: July 17, 2018

Accepted: October 7, 2018

Published: October 10, 2018

Copyright $\odot 2018$ by authors and Scientific Research Publishing Inc. This work is licensed under the Creative Commons Attribution International License (CC BY 4.0).

http://creativecommons.org/licenses/by/4.0/

\begin{abstract}
The aim of the present work is to study the complete, vertical and horizontal lifts using Tachibana and Visknnevskii operators along generalized almost $\mathrm{r}$-contact structure in tangent bundle. We also prove certain theorems on Tachibana and Visknnevskii operators with Lie derivative and lifts.
\end{abstract}

\section{Keywords}

Tangent Bundle, Vertical Lift, Complete Lift, Lie Derivative, Tachibana Operator, Vishnevskii Operator

\section{Introduction}

\section{Open Access}

be its tangent bundle. Then $T(M)$ is also a differentiable manifold [1]. Let $X=\sum_{i=1}^{n} x^{i}\left(\frac{\partial}{\partial x^{i}}\right)$ and $\eta=\sum_{i=1}^{n} \eta^{i} d x^{i}$ be the expressions in local coordinates for the vector field $X$ and the 1-form $\eta$ in $M$. Let $\left(x^{i}, y^{i}\right)$ be local coordinates of point in $T(M)$ induced naturally from the coordinate chart $\left(U, x^{i}\right)$ in $M$.

The complete, vertical and horizontal lifts of tensor field have vital role in differential geometry of tangent bundle. In 2016, [2] studied Tachibana and Vishneveskii operators applied to vertical and horizontal lifts in almost paracontact structure on the tangent bundle $T(M)$. The generalized almost $r$-contact structure in tangent bundle and integrability of structure is studied by the second author [3]. 
This paper is organized as follows: Section 2 describes some basic definitions and notations. Section 3 deals with the study of Tachibana and Vishnevskii operators for generalized almost $r$-contact structure in tangent bundle.

\section{Preliminaries}

\subsection{Vertical Lifts}

If $f$ is a function in $M$, we write $f^{V}$ for the function in $T(M)$ obtained by forming the composition of $\pi: T(M) \rightarrow M$ and $f: M \rightarrow R$, so that

$$
f^{V}=f \circ \pi
$$

where $\circ$ is composition of $f$ and $p i$.

Thus, if a point $\tilde{p} \in \pi^{-1}(U)$ has induced coordinates $\left(x^{h}, y^{h}\right)$ then

$$
f^{V}(\tilde{p})=f^{V}(x, y)=f \circ \pi(\tilde{p})=f(p)=f(x)
$$

Thus the value of $f^{V}(\tilde{p})$ is constant along each fibre $T_{p}(M)$ and equal to the value $f(p)$. We call $f^{V}$ the vertical lift of the function $f$.

Vertical lifts to a unique algebraic isomorphism of the tensor algebra $\mathfrak{I}(M)$ into the tensor algebra $\mathfrak{I}(T(M))$ with respect to constant coefficients by the conditions (Tensor product of $P$ and $Q$ )

$$
(P \otimes Q)^{V}=P^{V} \otimes Q^{V},(P+R)^{V}=P^{V}+R^{V}
$$

$P, Q$ and $R$ being arbitrary elements of $\mathfrak{I}(M)$.

Furthermore, the vertical lifts of tensor fields obey the general properties [4] [5]:

(a) $(f \cdot g)^{V}=f^{V} g^{V},(f+g)^{V}=f^{V}+g^{V}$,

(b) $(X+Y)^{V}=X^{V}+Y^{V},(f \cdot X)^{V}=f^{V} X^{V}, X^{V} f^{V}=0,\left[X^{V}, Y^{V}\right]=0$,

(c) $(f \cdot \eta)^{V}=f^{V} \eta^{V}, \eta^{V}\left(X^{V}\right)=0, X^{V}\left(Y^{V}\right)=0$,

$$
\forall f, g \in \mathfrak{I}_{0}^{0}(M), X, Y \in \mathfrak{I}_{0}^{1}(M), \eta \in \mathfrak{I}_{1}^{0}(M) .
$$

\subsection{Complete Lifts}

If $f$ is a function in $M$, we write $f^{C}$ for the function in $T(M)$ defined by [1]

$$
f^{C}=i(d f)
$$

and call $f^{C}$ the complete lift of the function $f$. The complete lift $f^{C}$ of a function $f$ has the local expression

$$
f^{C}=y^{i} \partial_{i} f=\partial f
$$

with respect to the induced coordinates in $T(M)$, where $\partial f$ denotes $y^{i} \partial_{i} f$.

Suppose that $X \in \mathfrak{I}_{0}^{1}(M)$. We define a vector field $X^{C}$ in $T(M)$ by

$$
X^{C} f^{C}=(X f)^{C}
$$

$f$ being an arbitrary function in $M$ and call $X^{C}$ the complete lift of $X$ in $T(M)$. 
The complete lift $X^{C}$ of $X$ with components $x^{h}$ in $M$ has components

$$
X^{C}:\left[\begin{array}{c}
x^{h} \\
\partial x^{h}
\end{array}\right]
$$

with respect to the induced coordinates in $T(M)$.

Suppose that $\eta \in \mathfrak{I}_{0}^{1}(M)$ Then a 1 -form $\eta^{C}$ in $T(M)$ defined by

$$
\eta^{C}\left(X^{C}\right)=(\eta(X))^{C}
$$

$X$ being an arbitrary vector field in $M$. We call $\eta^{C}$ the complete lift of $\eta$.

The complete lifts to a unique algebra isomorphism of the tensor algebra $\mathfrak{I}(M)$ into the tensor algebra $\mathfrak{I}(T(M))$ with respect to constant coefficients, is given by the conditions

$$
(P \otimes Q)^{C}=P^{C} \otimes Q^{V}+P^{V} \otimes Q^{C},(P+R)^{C}=P^{C}+R^{C}
$$

$P, Q$ and $R$ being arbitrary elements of $\mathfrak{I}(M)$.

Moreover, the complete lifts of tensor fields obey the general properties [1] [4]:

(a) $(f X)^{C}=f^{C} X^{V}+f^{V} X^{C}=(X f)^{C}, X^{C} f^{V}=(X f)^{V}, X^{V} f^{C}=(X f)^{V}$,

(b) $\phi^{V} X C=(\phi X)^{V}, \phi^{C} X^{V}=(\phi X)^{V},(\phi X)^{C}=\phi^{C} X^{C}$,

(c) $\eta^{V} X^{C}=(\eta(X))^{C}, \eta^{C} X^{V}=(\eta(X))^{V}$,

(d) $\left[X^{V}, Y^{C}\right]=[X, Y]^{C}, I^{C}=I, I^{V} I^{C}=X^{V},\left[X^{C}, Y^{C}\right]=[X, Y]^{C}$

$$
\forall f, g \in \mathfrak{I}_{0}^{0}(M), X, Y \in \mathfrak{I}_{0}^{1}(M), \eta \in \mathfrak{I}_{1}^{0}(M) .
$$

\subsection{Horizontal Lifts}

The horizontal lift $f^{H}$ of $f \in \mathfrak{J}_{0}^{0}(M)$ to the tangent bundle $T(M)$ by

$$
(f)^{H}=f^{C}-\nabla_{\gamma} f
$$

where

$$
\nabla_{\gamma} f=\gamma(\nabla f)
$$

Let $X \in \mathfrak{I}_{0}^{1}(M)$. Then the horizontal lift $X^{H}$ of $X$ defined by

$$
X^{H}=X^{C}-\nabla_{\gamma} X
$$

in $T(M)$, where

$$
\nabla_{\gamma} X=\gamma(\nabla X)
$$

The horizontal lift $X^{H}$ of $X$ has the components

$$
\left[\begin{array}{c}
x^{h} \\
-\Gamma_{i}^{h} x^{i}
\end{array}\right]
$$

with respect to the induced coordinates in $T(M)$, where $\Gamma_{i}^{h}=y^{j} \Gamma_{j i}^{h}$.

The horizontal lift $S^{H}$ of a tensor field $S$ of arbitrary type in $M$ to $T(M)$ is defined by

$$
S^{H}=S^{C}-\nabla_{\gamma} S
$$


for all $P, Q \in \mathfrak{I}(M)$. We have

$$
\nabla_{\gamma}(P \otimes Q)=\left(\nabla_{\gamma} P\right) \otimes Q^{V}+P^{V} \otimes\left(\nabla_{\gamma} Q\right)
$$

or

$$
(P \otimes Q)^{H}=P^{H} \otimes Q^{V}+P^{V} \otimes Q^{H} .
$$

In addition, the horizontal lifts of tensor fields obey the general properties [4] [6]:

(a) $X^{H} f^{V}=(X f)^{V}, \phi^{V} X^{H}=(\phi X)^{V}, \phi^{C} X^{H}=(\phi X)^{H}+\left(\nabla_{\gamma} \phi\right) X^{H}$;

(b) $\eta^{V}\left(X^{H}\right)=(\eta(X))^{H}, \eta^{C}\left(X^{H}\right)=(\eta(X))^{C}-\gamma(\eta \circ(\nabla X))$;

(c) $\eta^{H}\left(X^{C}\right)=\eta^{H}\left(\nabla_{\gamma} X\right), \eta^{H}\left(X^{H}\right)=0$

$$
\forall f, g \in \mathfrak{I}_{0}^{0}(M), X, Y \in \mathfrak{I}_{0}^{1}(M), \eta \in \mathfrak{I}_{1}^{0}(M), \phi \in \mathfrak{I}_{1}^{1}(M) .
$$

Let $X$ be a vector field in an n-dimensional differentiable manifold $M$. The differential transformation $L_{X}$ is called Lie derivative with respect to $X$ if

(a) $L_{X} f=X f, \forall f \in \mathfrak{J}_{0}^{0}(M)$,

(b) $L_{X} Y=[X, Y], \forall X, Y \in \mathfrak{I}_{0}^{1}(M)$.

The Lie derivative $L_{X} F$ of a tensor field $F$ of type $(1,1)$ with respect to a vector field $X$ is defined by

$$
\left(L_{X} F\right)=[X, F Y]-F[X, Y]
$$

where [,] is Lie bracket [1] page 113.

Let $M$ be an n-dimensional differentiable manifold. Differential transformation of algebra $T(M)$ defined by

$$
D=\nabla_{X}: T(M) \rightarrow T(M), X \in \mathfrak{I}_{0}^{1}(M),
$$

is called as covariant derivation with respect to vector field $X$ if

(a) $\nabla_{f X+g Y} t=f \nabla_{X} t+g \nabla_{Y} t$,

(b) $\nabla_{X} f=X f$,

$$
\forall f, g \in \mathfrak{J}_{0}^{0}(M), \forall X, Y \in \mathfrak{I}_{0}^{1}(M), \forall t \in \mathfrak{I}(M) .
$$

and a transformation defined by

$$
\nabla: \mathfrak{I}_{0}^{1}(M) \times \mathfrak{I}_{0}^{1}(M) \rightarrow \mathfrak{I}_{0}^{1}(M)
$$

is called affine connection [1].

Proposition 1. For any $X, Y \in \mathfrak{J}_{0}^{1}(M)$ [4]

(a) $\left[X^{V}, Y^{H}\right]=[X, Y]^{V}-\left(\nabla_{X} Y\right)^{V}=-\left(\hat{\nabla}_{X} Y\right)^{V}$,

(b) $\left[X^{C}, Y^{H}\right]=[X, Y]^{H}-\gamma\left(L_{X} Y\right)$,

(c) $\left[X^{H}, Y^{V}\right]=[X, Y]^{V}+\left(\nabla_{Y} X\right)^{V}$,

(d) $\left[X^{C}, Y^{H}\right]=[X, Y]^{H}-\gamma \hat{R}(X, Y)$

where $\hat{R}$ denotes the curvature tensor of the affine connection $\hat{\nabla}$.

Proposition 2. For any $X, Y \in \mathfrak{I}_{0}^{1}(M), f \in \mathfrak{I}_{0}^{0}(M)$ and $\nabla^{H}$ is the 
horizontal lift of the affine connection $\nabla$ to $T(M)$
(a) $\nabla_{X^{V}}^{H} Y^{V}=0$
(b) $\nabla_{X^{V}}^{H} Y^{H}=0$,
(c) $\nabla_{X^{H}}^{H} Y^{V}=\left(\nabla_{X} Y\right)^{V}$,
(d) $\nabla_{X^{H}}^{H} Y^{H}=\left(\nabla_{X} Y\right)^{H}$.

\section{Tachibana and Vishnevskii Operators for Generalized Almost R-Contact Structure in Tangent Bundle}

Let $M$ be a differentiable manifold of $C^{\infty}$ class. Suppose that there are given a tensor field $\phi$ of type $(1,1)$, a vector field $\xi_{p}$ and a 1 -form $\eta_{p}, p=1,2, \cdots, r$ satisfying [7] [8] [9]
(a) $\phi^{2}=a^{2} I+\epsilon \sum_{p=1}^{r} \xi_{p} \otimes \eta_{p}$
(b) $\phi \xi_{p}=0$
(c) $\eta_{p} \circ \phi=0$
(d) $\eta_{p}\left(\xi_{q}\right)=-\frac{a^{2}}{\epsilon} \delta_{p q}$

where $p=1,2, \cdots, r$ and $\delta_{p q}$ denote the Kronecker delta while $a$ and $\epsilon$ are non-zero complex numbers. The manifold $M$ is called a generalized almost r-contact manifold with a generalized almost $r$-contact structure or in short with $\left(\phi, \eta_{p}, \xi_{p}, a, \epsilon\right)$-structure.

Let us suppose that the base space $M$ admits the Lorentzian almost r-para-contact structure. Then there exists a tensor field $\phi$ of type $(1,1)$, $r\left(C^{\infty}\right)$ vector fields $\xi_{1}, \xi_{2}, \cdots, \xi_{p}$ and $r\left(C^{\infty}\right)$ 1-forms $\eta_{1}, \eta_{2}, \cdots, \eta_{p}$ such that Equation (12) are satisfied. Taking complete lifts of Equation (12) we obtain the following:

$$
\begin{aligned}
& \text { (a) }\left(\phi^{H}\right)^{2}=a^{2} I+\epsilon \sum_{p=1}^{r}\left\{\xi_{p}^{V} \otimes \eta_{p}^{H}+\xi_{p}^{H} \otimes \eta_{p}^{V}\right\} \\
& \text { (b) } \phi^{H} \xi_{p}^{V}=0, \phi^{H} \xi_{p}^{C}=0 \\
& \text { (c) } \eta_{p}^{V} \circ \phi^{H}=0, \eta_{p}^{H} \circ \phi^{V}=0, \eta_{p}^{H} \circ \phi^{H}=0, \eta_{p}^{V} \circ \phi^{V}=0 \\
& \text { (d) } \eta_{p}^{H}\left(\xi_{p}^{H}\right)=\eta_{p}^{V}\left(\xi_{p}^{V}\right)=0, \eta_{p}^{H}\left(\xi_{p}^{V}\right)=\eta_{p}^{V}\left(\xi_{p}^{H}\right)=-\frac{a^{2}}{\epsilon} \delta_{p q}
\end{aligned}
$$

Let us define an element $\tilde{J}$ of $J_{0}^{1} T(M)$ by

$$
\tilde{J}=\phi^{H}+\frac{\epsilon}{a} \sum_{p=1}^{r}\left(\xi_{p}^{V} \otimes \eta_{p}^{V}+\xi_{p}^{H} \otimes \eta_{p}^{H}\right)
$$

then in the view of Equation (13), it is easily shown that

$$
\tilde{J}^{2} X^{V}=a^{2} X^{V}, \tilde{J}^{2} X^{H}=a^{2} X^{H}
$$

which givess that $\tilde{J}$ is GF structure in $T(M)$ [10].

Now in view of the Equation (15), we have 

(a) $\tilde{J} X^{H}=(\phi X)^{H}+\frac{\epsilon}{a} \sum_{p=1}^{r}\left\{\left(\eta_{p}(X)\right)^{V} \xi_{p}^{V}\right\}$
(b) $\tilde{J} X^{V}=(\phi X)^{V}+\frac{\epsilon}{a} \sum_{p=1}^{r}\left\{\left(\eta_{p}(X)\right)^{V} \xi_{p}^{H}\right\}$

for all $X \in \mathfrak{I}_{0}^{1}(M)$.

\subsection{Tachibana Operator}

Let $\phi$ be a tensor fieldof type $(1,1)$ i.e. $\phi \in \mathfrak{J}_{1}^{1}(M)$ and $\phi \in \mathfrak{J}(M)=\sum_{r, s=0}^{\infty} \mathfrak{\Im}_{r}^{s}(M)$ be a tensor algebra over $R$. A map $\left.\Phi_{\phi}\right|_{r+s>0}$ is called a Tachibana operator or $\Phi_{\phi}$ operator on $M$ if [2] [11]

(a) $\Phi_{\phi}$ is linear with respect to constant coefficient,

(b) $\Phi_{\phi}: \mathfrak{I}^{*}(M) \rightarrow \mathfrak{I}_{s+1}^{r}(M)$ for all $r$ and $s$,

(c) $\Phi_{\phi}\left(K \otimes^{C} L\right)=\left(\Phi_{\phi} K\right) \otimes L+K \otimes \Phi_{\phi} L$ for all $K, L \in \mathfrak{J}^{*}(M)$,

(d) $\Phi_{\phi X} Y=-\left(L_{Y} \phi\right) X$ for all $X, Y \in \mathfrak{I}_{0}^{1}(M)$

where $L_{Y}$ is Lie derivation with respect to $Y$,

$$
\text { (e) } \begin{aligned}
\left(\Phi_{\phi \eta}\right) Y & =\left(d \left(\mathfrak{\Im}_{Y} \eta(\Phi X)-\left(d \left(\mathfrak{\Im}_{Y}(\eta \circ \Phi) X+\eta\left(\left(L_{Y} \phi\right) X\right)\right.\right.\right.\right. \\
& =\left(\Phi X\left(\mathfrak{\Im}_{Y} \eta\right)\right)(\Phi X)-X\left(\mathfrak{J}_{\phi X} \eta\right)+\eta\left(\left(L_{Y} \phi\right) X\right)
\end{aligned}
$$

for all $\eta \in \mathfrak{I}_{1}^{0}(M)$ and $X, Y \in \mathfrak{I}_{0}^{1}(M)$, where $\mathfrak{I}_{Y} \eta=\eta(X)=\eta \otimes^{C} Y$, $\mathfrak{J}_{r}^{* s}(M)$ the module of pure tensor fields of type $(r, s)$ on $M$ with respect to the affinor field $\varphi$ [12] [13].

Theorem 3. For Tachibana operator on $M, L_{X}$ the operator Lie derivation with respect to $X, \tilde{J} \in \mathfrak{I}_{1}^{1}(T(M))$ defined by $\tilde{J}=\phi^{H}+\frac{\epsilon}{a} \sum_{p=1}^{r}\left(\xi_{p}^{V} \otimes \eta_{p}^{V}+\xi_{p}^{H} \otimes \eta_{p}^{H}\right)$ and $\eta(Y)=0$, we have

(a) $\Phi_{\tilde{J} Y^{V}} X^{H}=-\left(\left(\hat{\nabla}_{X} \phi\right) Y\right)^{V}+\frac{\epsilon}{a} \sum_{p=1}^{r}\left(\left(\hat{\nabla}_{X} \eta_{p}\right) Y\right)^{V} \xi_{p}^{H}$

(b) $\Phi_{\tilde{J}^{H}} X^{H}=-\left(\left(L_{X} \phi\right) Y\right)^{H}+\gamma \hat{R}(X, \phi Y)+\frac{\epsilon}{a} \sum_{p=1}^{r}\left(\left(L_{X} \eta_{p}\right) Y\right)^{V} \xi_{p}^{V}-\tilde{J} \gamma \hat{R}(X, Y)$

(c) $\Phi_{\tilde{J} Y^{V}} X^{V}=0$

(d)

$$
\begin{aligned}
\Phi_{\tilde{J} Y^{H}} X^{V}= & -\left(\left(L_{X} \phi\right) Y\right)^{V}+\left(\left(\nabla_{X} \phi\right) Y\right)^{V}-\frac{\epsilon}{a} \sum_{p=1}^{r}\left(\left(L_{X} \eta_{p}\right) Y\right)^{V} \xi_{p}^{H} \\
& +\frac{\epsilon}{a} \sum_{p=1}^{r}\left(\left(\nabla_{X} \eta_{p}\right) Y\right)^{V} \xi_{p}^{H}
\end{aligned}
$$

where $X, Y \in \mathfrak{I}_{0}^{1}(M)$, a tensor field $\phi \in \mathfrak{I}_{1}^{1}(M)$, a vector field $\xi$ and a 1-form $\eta \in \mathfrak{I}_{1}^{0}(M)$.

Proof. For $\tilde{J}=\phi^{H}+\frac{\epsilon}{a} \sum_{p=1}^{r}\left(\xi_{p}^{V} \otimes \eta_{p}^{V}+\xi_{p}^{H} \otimes \eta_{p}^{H}\right)$ and $\eta(Y)=0$, we get 


$$
\begin{aligned}
& \Phi_{\tilde{J} Y^{V}} X^{H}=-\left(L_{X^{H}} \tilde{J}\right) Y^{V}=-\left(L_{X^{H}} \tilde{J} Y^{V}-\tilde{J} L_{X^{H}} Y^{V}\right) \text {, since } L_{X} Y=[X, Y] \\
& =-\left[X^{H}, \tilde{J} Y^{V}\right]+\left(\phi^{H}+\frac{\epsilon}{a} \sum_{p=1}^{r}\left(\xi_{p}^{V} \otimes \eta_{p}^{V}+\xi_{p}^{H} \otimes \eta_{p}^{H}\right)\right)\left[X^{H}, Y^{V}\right] \\
& \text { (a) }=-\left[X^{H},(\phi Y)^{V}\right]+\phi^{H}\left([X, Y]^{V}+\left(\nabla_{X} Y\right)^{V}\right) \\
& +\frac{\epsilon}{a} \sum_{p=1}^{r} \eta_{p}^{V}\left([X, Y]^{V}+\left(\nabla_{Y} X\right)^{V}\right) \xi_{p}^{V}+\frac{\epsilon}{a} \sum_{p=1}^{r} \eta_{p}^{H}\left([X, Y]^{V}+\left(\nabla_{Y} X\right)^{V}\right) \xi_{p}^{H} \\
& =-\left[X^{H},(\phi Y)^{V}\right]\left(\nabla_{\phi Y} X\right)^{V}+\phi^{H}\left([X, Y]^{V}+\left(\nabla_{Y} X\right)^{V}\right) \\
& +\frac{\epsilon}{a} \sum_{p=1}^{r} \eta_{p}^{V}\left([X, Y]^{V}+\left(\nabla_{Y} X\right)^{V}\right) \xi_{p}^{V}+\frac{\epsilon}{a} \sum_{p=1}^{r} \eta_{p}^{H}\left([X, Y]^{V}+\left(\nabla_{Y} X\right)^{V}\right) \xi_{p}^{H} \\
& =-\left(\left(\hat{\nabla}_{X} \phi\right) Y\right)^{V}-\left(\phi \hat{\nabla}_{X} Y\right)^{V}+\left(\phi\left(\hat{\nabla}_{X} Y\right)\right)^{V}+\frac{\epsilon}{a} \sum_{p=1}^{r}\left(\left(L_{X} \eta_{p}\right) Y\right)^{V} \xi_{p}^{H} \\
& -\frac{\epsilon}{a} \sum_{p=1}^{r}\left(\left(\hat{\nabla}_{X} \eta_{p}\right) Y\right)^{V} \xi_{p}^{H}-\frac{\epsilon}{a} \sum_{p=1}^{r}\left(\eta_{p}\left(L_{X} Y\right)\right)^{V} \xi_{p}^{H} \\
& \text { as } \eta\left(L_{X} Y\right)=-\left(L_{X} \eta_{p}\right) Y=-\left(\left(\hat{\nabla}_{X} \phi\right) Y\right)^{V}-\frac{\epsilon}{a} \sum_{p=1}^{r}\left(\left(\hat{\nabla}_{X} \eta_{p}\right) Y\right)^{V} \xi_{p}^{H} \text {. } \\
& \Phi_{\tilde{J} Y^{H}} X^{H}=-\left(L_{X^{H}} \tilde{J}\right) Y^{H}=-\left(L_{X^{H}} \tilde{J} Y^{H}-\tilde{J} L_{X^{H}} Y^{H}\right) \text { since } L_{X} Y=[X, Y] \\
& =-\left[X^{H}, \tilde{J} Y^{H}\right]+\left(\phi^{H}+\frac{\epsilon}{a} \sum_{p=1}^{r}\left(\xi_{p}^{V} \otimes \eta_{p}^{V}+\xi_{p}^{H} \otimes \eta_{p}^{H}\right)\right)\left[X^{H}, Y^{H}\right] \\
& \text { (b) }=-\left[X^{H},(\phi Y)^{H}\right]+\phi^{H}\left[X^{H}, Y^{H}\right]+\frac{\epsilon}{a} \sum_{p=1}^{r} \eta_{p}^{V}\left[X^{H}, Y^{H}\right] \xi_{p}^{V}+\frac{\epsilon}{a} \sum_{p=1}^{r} \eta_{p}^{H}\left[X^{H}, Y^{H}\right] \xi_{p}^{H} \\
& \text { since }\left[X^{H}, Y^{H}\right]=[X, Y]^{H}-\gamma \hat{R}(X, Y) \text {, } \\
& =-\left(\left(L_{X} \phi\right) Y\right)^{H}+\gamma \hat{R}(X, \phi Y)-\frac{\epsilon}{a} \sum_{p=1}^{r}\left(\left(L_{X} \eta_{p}\right) Y\right)^{V} \xi_{p}^{V}-\tilde{J} \gamma \hat{R}(X, Y) \text {. } \\
& \Phi_{\tilde{J} Y^{V}} X^{V}=-\left(L_{X^{V}} \tilde{J}\right) Y^{V}=-\left(L_{X^{V}} \tilde{J} Y^{V}-\tilde{J} L_{X^{V}} Y^{V}\right) \text { since } L_{X} Y=[X, Y] \\
& =-\left[X^{V}, \tilde{J} Y^{V}\right]+\tilde{J}\left[X^{V}, Y^{V}\right], \quad\left[X^{V}, Y^{V}\right]=0 \\
& \text { (c) }=-\left[X^{V},\left(\phi^{H}+\frac{\epsilon}{a} \sum_{p=1}^{r}\left(\xi_{p}^{V} \otimes \eta_{p}^{V}+\xi_{p}^{H} \otimes \eta_{p}^{H}\right)\right) Y^{V}\right] \\
& \text { as }\left(\eta_{p}(Y) \xi_{p}\right)^{H}=0=-\left[X^{V},(\phi Y)^{V}\right]-\frac{\epsilon}{a} \sum_{p=1}^{r}\left[X^{V},\left(\eta_{p}(Y) \xi_{p}\right)^{H}\right]=0 . \\
& \Phi_{\tilde{J}^{H}} X^{V}=-\left(L_{X^{V}} \tilde{J}\right) Y^{H}=-L_{X^{V}} \tilde{J} Y^{H}+\tilde{J} L_{X^{V}} Y^{H} \text {, since } L_{X} Y=[X, Y] \\
& =-\left[X^{V}, \tilde{J} Y^{H}\right]+\left(\phi^{H}+\frac{\epsilon}{a} \sum_{p=1}^{r}\left(\xi_{p}^{V} \otimes \eta_{p}^{V}+\xi_{p}^{H} \otimes \eta_{p}^{H}\right)\right)\left[X^{V}, Y^{H}\right] \\
& =-[X, \phi Y]^{V}+\left(\nabla_{X} \phi Y\right)^{V}+\phi^{H}\left([X, Y]^{V}-\left(\nabla_{X} Y\right)^{V}\right) \\
& \text { (d) } \\
& +\frac{\epsilon}{a} \sum_{p=1}^{r} \eta_{p}^{V}\left([X, Y]^{V}-\left(\nabla_{X} Y\right)^{V}\right) \xi_{p}^{V}+\frac{\epsilon}{a} \sum_{p=1}^{r} \eta_{p}^{H}\left([X, Y]^{V}-\left(\nabla_{X} Y\right)^{V}\right) \xi_{p}^{H} \\
& \text { since } \eta_{p} L_{X} Y=L_{X} \eta_{p}(Y)-\left(L_{X} \eta_{p}\right) Y, \eta_{p} \nabla_{X} Y=\nabla_{X} \eta_{p}(Y)-\left(\nabla_{X} \eta_{p}\right) Y \\
& =-\left(\left(L_{X} \phi\right) Y\right)^{V}+\left(\left(\nabla_{X} \phi\right) Y\right)^{V}-\frac{\epsilon}{a} \sum_{p=1}^{r}\left(\left(L_{X} \eta_{p}\right) Y\right)^{V} \xi_{p}^{H}+\frac{\epsilon}{a} \sum_{p=1}^{r}\left(\left(\nabla_{X} \eta_{p}\right) Y\right)^{V} \xi_{p}^{H} \text {. }
\end{aligned}
$$


Corollary 1. If we put $Y=\xi_{p}$ i.e. $\eta_{p}^{H}\left(\xi_{p}^{H}\right)=\eta_{p}^{V}\left(\xi_{p}^{V}\right)=0$, $\eta_{p}^{H}\left(\xi_{p}^{V}\right)=\eta_{p}^{V}\left(\xi_{p}^{H}\right)=-\frac{a^{2}}{\epsilon}$, then we have

(a) $\Phi_{\tilde{J} \xi_{p}^{V}} X^{H}=a \sum_{p=1}^{r}\left(L_{\xi_{p}} X\right)^{H}-a \gamma \hat{R}\left(X, \xi_{p}\right)-\left(\left(\hat{\nabla}_{X} \phi\right) \xi_{p}\right)^{V}+\left(\left(\hat{\nabla}_{X} \eta_{p}\right) \xi_{p}\right)^{V} \xi_{p}^{H}$

(b)

$$
\begin{aligned}
\Phi_{\tilde{J}_{\xi_{p}^{H}}} X^{H}= & a\left(\hat{\nabla}_{X} \xi_{p}\right)^{V}-\left(\left(L_{X} \phi\right) \xi_{p}\right)^{H}-\phi^{H} \gamma \hat{R}\left(X, \xi_{p}\right)-\frac{\epsilon}{a} \sum_{p=1}^{r}\left(\left(L_{X} \eta_{p}\right) \xi_{p}\right)^{V} \xi_{p}^{V} \\
& -\frac{\epsilon}{a} \sum_{p=1}^{r} \eta_{p}^{V} \gamma \hat{R}\left(X, \xi_{p}\right) \xi_{p}^{V}-\frac{\epsilon}{a} \sum_{p=1}^{r} \eta_{p}^{H} \gamma \hat{R}\left(X, \xi_{p}\right) \xi_{p}^{H} .
\end{aligned}
$$

(c) $\Phi_{\tilde{J} \xi_{p}^{V}} X^{V}=-a\left(\hat{\nabla}_{\xi_{p}} X\right)^{V}$

(d)

$$
\begin{aligned}
\Phi_{\tilde{J} \xi_{p}^{H}} X^{V}= & -\left(\left(L_{X} \phi\right) \xi_{p}\right)^{V}+\left(\left(\nabla_{X} \phi\right) \xi_{p}\right)^{V}-\frac{\epsilon}{a} \sum_{p=1}^{r}\left(\left(L_{X} \eta_{p}\right) \xi_{p}\right)^{V} \xi_{p}^{H} \\
& +\frac{\epsilon}{a} \sum_{p=1}^{r}\left(\left(\nabla_{X} \eta_{p}\right) \xi_{p}\right)^{V} \xi_{p}^{H} .
\end{aligned}
$$

\subsection{Vishnevskii Operator}

Let $\nabla$ is a linear connection and $\phi$ be a tensor field of type $(1,1)$ on $M$. If the condition (d) of Tachibana operator replace by

$$
\text { (d') } \Psi_{\phi X} Y=\nabla_{\phi X} Y-\phi \nabla_{X} Y,
$$

$\forall X, Y \in \mathfrak{I}_{0}^{1}(M)$, is a mapping wih linear connection $\nabla$. A map $\Psi_{\phi}: \mathfrak{J}^{*}(M) \rightarrow \mathfrak{I}(M)$, which satisfies conditions (a), (b), (c), (e) of Tachibana operator and the condition (d'), is called Vishnevskii operator on $M$ [2] [14].

Theorem 4. For $\Psi_{\phi} \quad$ Vishnevskii operator on $M$ and $\nabla^{H}$ the horizontal lift of an affine connection $\nabla$ in $M$ to $T(M), \tilde{J} \in \mathfrak{J}_{1}^{1}(T(M))$ defined by (14), we have

(a) $\Psi_{\tilde{J} X^{V}} Y^{H}=\frac{\epsilon}{a} \sum_{p=1}^{r}\left(\eta_{p}(X) \nabla_{\xi p} Y\right)^{H}$

(b)

$$
\begin{aligned}
\Psi_{\tilde{J} X^{H}} Y^{V}= & \left(\left(\hat{\nabla}_{Y} \phi\right) X\right)^{V}-\left(\left(L_{X} \phi\right) X\right)^{V}-\frac{\epsilon}{a} \sum_{p=1}^{r}\left(\eta_{p} \hat{\nabla}_{Y} X\right)^{V} \xi_{p}^{H} \\
& +\frac{\epsilon}{a} \sum_{p=1}^{r}\left(\eta_{p} L_{Y} X\right)^{V} \xi_{p}^{H}
\end{aligned}
$$

(c) $\Psi_{\tilde{J} X^{V}} Y^{V}=\frac{\epsilon}{a} \sum_{p=1}^{r}\left(\eta_{p}(X)\right)^{V} \nabla_{\xi_{p}^{H}}^{H} Y^{V}$

(d)

$$
\begin{aligned}
\Psi_{\tilde{J} X} Y^{H}= & \left(\left(\hat{\nabla}_{Y} \phi\right) X\right)^{H}-\left(\left(L_{X} \phi\right) X\right)^{H}-\frac{\epsilon}{a} \sum_{p=1}^{r}\left(\eta_{p} \hat{\nabla}_{Y} X\right)^{V} \xi_{p}^{V} \\
& +\frac{\epsilon}{a} \sum_{p=1}^{r}\left(\eta_{p} L_{Y} X\right)^{V} \xi_{p}^{V}
\end{aligned}
$$

where $X, Y \in \mathfrak{I}_{0}^{1}(M)$, a tensor field $\phi \in \mathfrak{J}_{1}^{1}(M)$, vector fields $\xi_{p}$ and a 1-form $\eta_{p} \in \mathfrak{I}_{1}^{0}(M), p=1, \cdots, r$.

Proof. 


$$
\begin{aligned}
& \Psi_{\tilde{J} X^{V}} Y^{H}=\nabla_{\tilde{J} X^{V}}^{H} Y^{H}-\tilde{J} \nabla_{X^{V}}^{H} Y^{H} \\
& =\nabla_{\left(\phi^{H}+\frac{\epsilon}{a} \sum_{p=1}^{r}\left(\xi_{p}^{V} \otimes \eta_{p}^{V}+\xi_{p}^{H} \otimes \eta_{p}^{H}\right)\right) X^{V}} Y^{H}-\left(\phi^{H}+\frac{\epsilon}{a} \sum_{p=1}^{r}\left(\xi_{p}^{V} \otimes \eta_{p}^{V}+\xi_{p}^{H} \otimes \eta_{p}^{H}\right)\right) \nabla_{X^{V}}^{H} Y^{H} \\
& \text { (a) }=\nabla_{(\phi X)^{V}+\frac{\epsilon}{a} \sum_{p=1}^{r}\left(\eta_{p} X\right)^{V} \xi_{p}^{H}} Y^{H} \text { as } \nabla_{X^{V}}^{H} Y^{H}=0 \\
& =\frac{\epsilon}{a} \sum_{p=1}^{r}\left(\eta_{p} X\right)^{V}\left(\nabla_{\xi_{p}} Y\right)^{H} \text { as } \nabla_{(\phi X)}^{H} Y^{H}=0 \\
& =\frac{\epsilon}{a} \sum_{p=1}^{r}\left(\eta_{p}(X) \nabla_{\xi_{p}} Y\right)^{H} \text {. } \\
& \Psi_{\tilde{J} X^{H}} Y^{V}=\nabla_{\tilde{J} X^{H}}^{H} Y^{H}-\tilde{J} \nabla_{X^{H}}^{H} Y^{V} \\
& =\nabla_{\left(\phi^{H}+\frac{\epsilon}{a} \sum_{p=1}^{r}\left(\xi_{p}^{V} \otimes \eta_{p}^{V}+\xi_{p}^{H} \otimes \eta_{p}^{H}\right)\right) X^{H}}^{H} Y^{V}-\left(\phi^{H}+\frac{\epsilon}{a} \sum_{p=1}^{r}\left(\xi_{p}^{V} \otimes \eta_{p}^{V}+\xi_{p}^{H} \otimes \eta_{p}^{H}\right)\right) \nabla_{X^{H}}^{H} Y^{V} \\
& =\nabla_{(\phi X)^{H}}^{H} Y^{V}-\phi^{H}\left(\nabla_{X} Y\right)^{V}-\frac{\epsilon}{a} \sum_{p=1}^{r} \eta_{p}^{H}\left(\nabla_{X} Y\right)^{V} \xi_{p}^{H} \\
& \text { (b) } \\
& =\left(\hat{\nabla}_{Y} \phi X\right)^{V}+[\phi X, Y]^{V}-\phi^{H}\left(\left(\hat{\nabla}_{Y} X\right)^{V}+[X, Y]^{V}\right) \\
& -\frac{\epsilon}{a} \sum_{p=1}^{r} \eta_{p}^{H}\left(\left(\hat{\nabla}_{Y} X\right)^{V}+[X, Y]^{V}\right) \xi_{p}^{H} \\
& =\left(\left(\hat{\nabla}_{Y} \phi\right) X\right)^{V}-\left(\left(L_{Y} \phi\right) X\right)^{V}-\frac{\epsilon}{a} \sum_{p=1}^{r}\left(\eta_{p} \hat{\nabla}_{Y} X\right)^{V} \xi_{p}^{H}+\frac{\epsilon}{a} \sum_{p=1}^{r}\left(\eta_{p} L_{Y} X\right)^{V} \xi_{p}^{H} \\
& \Psi_{\tilde{J} X^{V}} Y^{V}=\nabla_{\tilde{J} X^{V}}^{H} Y^{V}-\tilde{J} \nabla_{X^{V}}^{H} Y^{V} \\
& =\nabla_{\left(\phi^{H}+\frac{\epsilon}{a} \sum_{p=1}^{r}\left(\xi_{p}^{V} \otimes \eta_{p}^{V}+\xi_{p}^{H} \otimes \eta_{p}^{H}\right)\right) X^{V}}^{H} Y^{V}-\left(\phi^{H}+\frac{\epsilon}{a} \sum_{p=1}^{r}\left(\xi_{p}^{V} \otimes \eta_{p}^{V}+\xi_{p}^{H} \otimes \eta_{p}^{H}\right)\right) \nabla_{X^{V}}^{H} Y^{V} \\
& \text { (c) } \\
& =\nabla_{(\phi X)}^{H} Y^{V}+\frac{\varepsilon}{a} \sum_{p=1}^{r}\left(\eta_{p}(X)\right)^{V} \nabla_{\xi_{p}^{H}}^{H} Y^{V} \\
& =\frac{\epsilon}{a} \sum_{p=1}^{r}\left(\eta_{p}(X)\right)^{V} \nabla_{\xi_{p}^{H}}^{H} Y^{V} \quad \text { as } \nabla_{(\phi X)}^{H} Y^{V}=0 . \\
& \Psi_{\tilde{J} X^{H}} Y^{H}=\nabla_{\tilde{J} X^{H}}^{H} Y^{H}-\tilde{J} \nabla_{X^{H}}^{H} Y^{H} \\
& =\nabla_{\left(\phi^{H}+\frac{\epsilon}{a} \sum_{p=1}^{r}\left(\xi_{p}^{V} \otimes \eta_{p}^{V}+\xi_{p}^{H} \otimes \eta_{p}^{H}\right)\right) X^{H}} Y^{H}-\left(\phi^{H}+\frac{\epsilon}{a} \sum_{p=1}^{r}\left(\xi_{p}^{V} \otimes \eta_{p}^{V}+\xi_{p}^{H} \otimes \eta_{p}^{H}\right)\right) \nabla_{X^{H}}^{H} Y^{H} \\
& =\nabla_{(\phi X)}^{H} Y^{H}-\phi^{H}\left(\nabla_{X} Y\right)^{H}-\frac{\epsilon}{a} \sum_{p=1}^{r} \eta_{p}^{V}\left(\nabla_{X} Y\right)^{H} \xi_{p}^{V} \\
& \text { (d) } \\
& =\left(\hat{\nabla}_{Y} \phi X\right)^{H}+[\phi X, Y]^{H}-\phi^{H}\left(\left(\hat{\nabla}_{Y} X\right)^{H}+[X, Y]^{H}\right) \\
& -\frac{\epsilon}{a} \sum_{p=1}^{r} \eta_{p}^{V}\left(\left(\hat{\nabla}_{Y} X\right)^{H}+[X, Y]^{H}\right) \xi_{p}^{H} \\
& =\left(\left(\hat{\nabla}_{Y} \phi\right) X\right)^{H}-\left(\left(L_{Y} \phi\right) X\right)^{H}-\frac{\epsilon}{a} \sum_{p=1}^{r}\left(\eta_{p} \hat{\nabla}_{Y} X\right)^{V} \xi_{p}^{V}+\frac{\epsilon}{a} \sum_{p=1}^{r}\left(\eta_{p} L_{Y} X\right)^{V} \xi_{p}^{V}
\end{aligned}
$$

Corollary 2. If we put $Y=\xi_{p}$ i.e. $\eta_{p}^{H}\left(\xi_{p}^{H}\right)=\eta_{p}^{V}\left(\xi_{p}^{V}\right)=0$,

$\eta_{p}^{H}\left(\xi_{p}^{V}\right)=\eta_{p}^{V}\left(\xi_{p}^{H}\right)=-\frac{a^{2}}{\epsilon} \delta_{p q}$, then we have 
(a) $\Psi_{\tilde{J} \xi_{p}^{V}} Y^{H}=-a\left(\nabla_{\xi p} Y\right)^{H}$

(b)

$$
\begin{aligned}
\Psi_{\tilde{J} \xi_{p}^{H}} Y^{V}= & -\phi^{H}\left(\hat{\nabla}_{Y} \xi_{p}\right)^{V}-\left(\left(L_{Y} \phi\right) \xi_{p}\right)^{V}+\frac{\epsilon}{a} \sum_{p=1}^{r}\left(\eta_{p}\left(\hat{\nabla}_{Y} \xi_{p}\right)^{V}\right) \xi_{p}^{H} \\
& -\frac{\epsilon}{a} \sum_{p=1}^{r}\left(\eta_{p}\left(L_{Y} \xi_{p}\right)^{V}\right) \xi_{p}^{H}
\end{aligned}
$$

(c) $\Psi_{\tilde{J} \xi_{p}^{V}} Y^{V}=-a\left(\nabla_{\xi_{p}} Y\right)^{V}$

(d)

$$
\begin{aligned}
\Psi_{\tilde{J} \xi_{p}^{H}} Y^{H}= & \left(\left(\hat{\nabla}_{Y} \phi\right) \xi_{p}\right)^{H}-\left(\left(L_{Y} \phi\right) \xi_{p}\right)^{H}+\frac{\epsilon}{a} \sum_{p=1}^{r}\left(\left(\hat{\nabla}_{Y} \eta_{p}\right) \xi_{p}\right)^{V} \xi_{p}^{V} \\
& -\frac{\epsilon}{a} \sum_{p=1}^{r}\left(\left(L_{Y} \eta_{p}\right) \xi_{p}\right)^{V} \xi_{p}^{V} .
\end{aligned}
$$

\section{Conclusion}

The generalized almost $\mathrm{r}$-contact structure on Tachibana and Visknnevskii operators in tangent bundle are introduced. We deduced the theorems on Tachibana and Visknnevskii operators with respect to Lie derivative and lifting theory.

\section{Acknowledgements}

We thank the Editor and the referee for their comments.

\section{Conflicts of Interest}

The authors declare no conflicts of interest regarding the publication of this paper.

\section{References}

[1] Yano, K. and Ishihara, S. (1973) Tangent and Cotangent Bundles. Marcel Dekker. Inc. New York.

[2] Cayir, H. (2016) Tachibana and Vishnevskii Operators Applied to $X^{V}$ and $X^{H}$ in Almost Paracontact Structure on Tangent Bundle $T(M)$. New Trends in Mathematical Sciences, 4, 105-115. https://doi.org/10.20852/ntmsci.2016318821

[3] Khan, M.N.I. (2017) A Note on Certain Structures in the Tangent Bundle. Far East Journal of Mathematical Sciences, 101, 1947-1965.

[4] Omran, T., Sharffuddin, A. and Husain, S.I. (1984) Lift of Structures on Manifold. Publications De L' institut Mathmatique (Beograd) (N.S.), 36, 93-97.

[5] Khan, M.N.I. (2014) Lifts of Hypersurfaces with Quarter-Symmetric Semi-Metric Connection to Tangent Bundles. Afrika Matematika, 25, 475-482. https://doi.org/10.1007/s13370-013-0150-x

[6] Das, L.S. and Khan, M.N.I. (2005) Almost R-Contact Structures on the Tangent Bundle. Differential Geometry-Dynamical Systems, 7, 34-41.

[7] Das, L.S. and Nivas, R. (2006) On Certain Structures Defined on the Tangent Bundle. Rocky Mountain Journal of Mathematics, 36, 1857-1866. https://doi.org/10.1216/rmjm/1181069348

[8] Vanzura, J. (1970) Almost R-Contact Structure. Annali Della Scuola Normale, Su- 
periore Di Pisa, 26, 97-115.

[9] Blair, D.E. (1976) Contact Manifolds in Riemannian Geometry. Vol. 509, Springer Verlag, New York. https://doi.org/10.1007/BFb0079307

[10] Das, L.S., Nivas, R. and Khan, M.N.I. (2009) On Submanifolds of Co-Dimension 2 Immersed in a Hsuquaternion Manifold. Acta Mathematica Academiae Paedagogicae Nyregyhziensis, 25, 129-135.

[11] Magden, A. (2004) On Applications of the Tachibana Operator. Applied Mathematics and Computation, 147, 45-55.

https://doi.org/10.1016/S0096-3003(02)00649-5

[12] Tekkoyun, M. (2006) On Lifts of Paracomplex Structures. Turkish Journal of Math, 30, 197-210.

[13] Khan, M.N.I. (2016) Lift of Semi-Symmetric Non-Metric Connection on a Khler Manifold. Afrika Matematika, 27, 345-352. https://doi.org/10.1007/s13370-015-0350-7

[14] Salimov, A.A. (2013) Tensor Operators and Their Applications. Nova Science Publ. New York. 